\title{
Editorial: Signaling by Small GTPases in Metastatic Disease
}

\author{
Peter J. Parker ${ }^{1 *}$ and Uday Kishore ${ }^{2 *}$ \\ ${ }^{1}$ Francis Crick Institute and King's College London, London, United Kingdom, ${ }^{2}$ Biosciences, Brunel University London, Uxbridge, \\ United Kingdom
}

Keywords: small GTPase, metastatic disease, cancer, superfamily, signalling

Editorial on the Research Topic

Signaling by Small GTPases in Metastatic Disease

The superfamily of small GTPases are allosteric regulators par excellence, touching almost all corners of mammalian cell biology (Reiner and Lundquist, 2018). These GTPases sit on regulatory pathways impacting amongst other processes: membrane traffic, nuclear transport, cell division and cytoskeletal organisation. Their downstream effectors embrace a spectrum of functions from second messenger generators and protein kinases to compartmentalised binding platforms and transport chaperones. Given this breadth of action, it is perhaps not surprising that several family members have been implicated in the genesis and dissemination of cancers as exemplified by the Rho subfamily (Vega and Ridley, 2008).

Structurally, this family of GTPases retain a conserved core associated with their nucleotide binding function and for most superfamily members, but not all, there is an intrinsic GTPase activity which contributes to their dynamic behaviour switching from active (GTP bound) to inactive (GDP bound) states. Physiologically this switch is not a protein autonomous function governed simply by the GTP/GDP ratio, rather it is facilitated by exchange factors that unload GDP and enable reloading from the GTP dominated guanine nucleotide pool (Toma-Fukai and Shimizu, 2019), and GTPase-activating proteins that determine the rate of bound GTP hydrolysis and hence inactivation (Mishra and Lambright, 2016).

For the Ras subfamily of GTPases there has been long-standing interest in their driver roles in cancer, reflecting the penetrance of somatic gain-of-function mutations which occur in a breadth of different cancers. This has engendered a substantial literature on the targeting of these proteins (Moore et al., 2020) and it is not the intention to recapitulate this here. Rather this volume focuses on the wider membership of this class of regulators, where penetrant mutations are not generally associated with the GTPases themselves, rather involvement in cancer is evidenced by a combination of changes in expression, dysregulation of GTPase regulators (mutation and/or expression) and a priori consideration of specific roles for members of this family [see for example the Rho family (Haga and Ridley, 2016)]. Metastatic spread of disease is the major cause of mortality in cancer and it is this process in particular that is the focus of attention here. A multistep process that requires migration through basement membrane, intravasation into the circulation, extravasation to tissues and ultimately the lodging of the tumour in secondary sites (Madsen and Sahai, 2010). The extensive movement of tumour cells through "hostile" environments selects for many distinctive attributes amongst which is a demand on the reorganisation of the cytoskeleton, a well-established target of small GTPase action. Might intervention in these processes limit dissemination and the associated mortality?

Targeting these proteins is not trivial despite the presence of a druggable nucleotide binding pocket. This is largely the consequence of the very high affinity for GTP that generally precludes pocket-binding competitive inhibitors acting with any potency; the covalent inhibitors targeting the cys-mutant of Ras present an interesting exception to this generality (Lim et al., 2014). Alternative pockets on these proteins offer some opportunities, but their regulators, in particular the exchange factors, and some downstream effectors (e.g., protein kinases) have proven more tractable drugging challenges [for example, the PAKs 
(Semenova and Chernoff, 2017)]. Understanding these pathways, therefore, constitutes an important element in any attack on these processes.

In this volume, invited contributors review various aspects of the actions of some key representatives of the small GTPase superfamily. The validation of these as targets, their direct or indirect targeting and the potential for utility in the clinic are discussed. It is evident that much progress has been made in specific approaches to this general problem of intervention but nevertheless there is a great deal to do in exploiting our knowledge of these proteins to the benefit of patients. It is hoped that the articles in this volume will further stimulate efforts in this direction.

\section{OBITUARY}

\section{Dr Sunil Kumar Verma (1974-2021)}

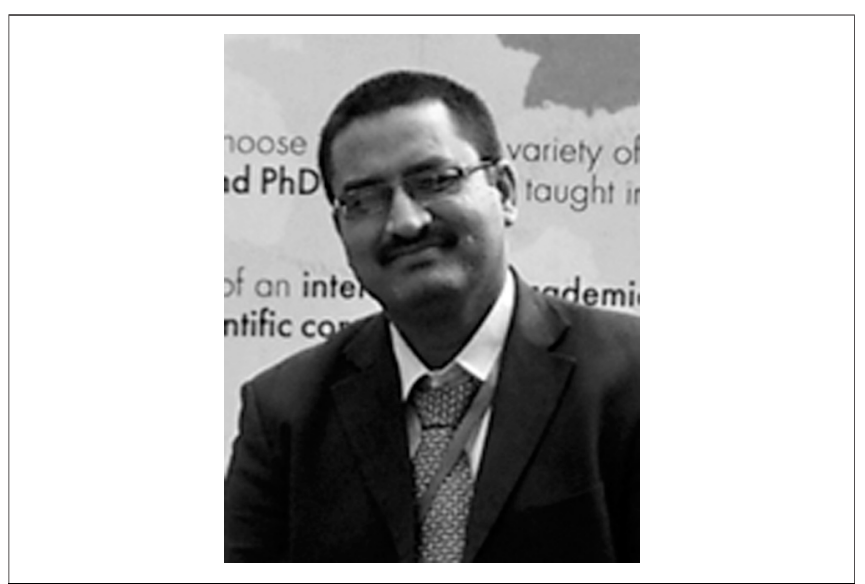

It is with great sadness that we mourn the death of Dr Sunil Kumar Verma, who was the main Guest Editor of this special issue. Sunil contracted SARS-CoV-2 infection and subsequently passed away due to COVID-19 pneumonia on $31^{\text {st }}$ May 2021 in

\section{REFERENCES}

Haga, R. B., and Ridley, A. J. (2016). Rho GTPases: Regulation and Roles in Cancer Cell Biology. Small GTPases 7 (4), 207-221. doi:10.1080/21541248.2016. 1232583

Lim, S. M., Westover, K. D., Ficarro, S. B., Harrison, R. A., Choi, H. G., Pacold, M. E., et al. (2014). Therapeutic Targeting of Oncogenic K-Ras by a Covalent Catalytic Site Inhibitor. Angew. Chem. Int. Ed. 53 (1), 199-204. doi:10.1002/ anie. 201307387

Madsen, C. D., and Sahai, E. (2010). Cancer Dissemination-Lessons from Leukocytes. Develop. Cel 19 (1), 13-26. doi:10.1016/j.devcel.2010.06.013

Mishra, A. K., and Lambright, D. G. (2016). Invited Review: Small GTPases and Their GAPs. Biopolymers 105 (8), 431-448. doi:10.1002/ bip. 22833

Moore, A. R., Rosenberg, S. C., McCormick, F., and Malek, S. (2020). RAS-targeted Therapies: Is the Undruggable Drugged. Nat. Rev. Drug Discov. 19 (8), 533-552. doi:10.1038/s41573-020-0068-6

Reiner, D. J., and Lundquist, E. A. (2018). Small GTPasesSmall GTPases. WormBook, 1-65. doi:10.1895/wormbook.1.67.2

Semenova, G., and Chernoff, J. (2017). Targeting PAK1. Biochem. Soc. Trans. 45 (1), 79-88. doi:10.1042/bst20160134 the Indian City of Hyderabad, where he worked as a Principal Scientist within the Centre for Cellular and Molecular Biology (Council for Scientific and Industrial Research).

We came to know and work with Sunil while he was doing his DPhil (PhD) degree in Medical Oncology from the Weatherall Institute of Molecular Medicine, University of Oxford. He also worked in the laboratory of Prof Peter Parker at the ICRF, London, UK during the latter half of his DPhil degree.

Sunil was a well-known scientific figure in India for his contributions to the development of a DNA barcoding method, in collaboration with his mentor, Prof Lalji Singh. This application is used to great effect in wildlife forensics. Sunil had a stellar career at the national level that brought him several prizes and awards, including CSIR Technology Award, NRDC Meritorious Invention Award, Emerging Forensic Scientist Continental Award, and BioAsia Innovation Award. He was also the recipient of Lindau Fellowship, Commonwealth Scholarship, Max Plank Visiting Fellowship, and DAAD ambassadorship.

Sunil grew up in a small village called Tikri in the Indian state of Uttar Pradesh. He studied his BSc in Agriculture and Food Technology from the GB Pant University. All through his life, he remained a very humble, honest and hardworking person. He also carried out a range of outreach activities including publishing a series of poems on India's topical issues.

Sunil will be sorely missed by his mentors, colleagues, family and friends. Rest in Peace!

Peter Parker, London, United Kingdom

Uday Kishore, London, United Kingdom

\section{AUTHOR CONTRIBUTIONS}

PP and UK drafted and edited jointly. Both authors approved the submitted version.

Toma-Fukai, S., and Shimizu, T. (2019). Structural Insights into the Regulation Mechanism of Small GTPases by GEFs. Molecules 24 (18). doi:10.3390/ molecules 24183308

Vega, F. M., and Ridley, A. J. (2008). Rho GTPases in Cancer Cell Biology. FEBS Lett. 582 (14), 2093-2101. doi:10.1016/j.febslet.2008.04.039

Conflict of Interest: The authors declare that the research was conducted in the absence of any commercial or financial relationships that could be construed as a potential conflict of interest.

Publisher's Note: All claims expressed in this article are solely those of the authors and do not necessarily represent those of their affiliated organizations, or those of the publisher, the editors and the reviewers. Any product that may be evaluated in this article, or claim that may be made by its manufacturer, is not guaranteed or endorsed by the publisher.

Copyright (c) 2022 Parker and Kishore. This is an open-access article distributed under the terms of the Creative Commons Attribution License (CC BY). The use, distribution or reproduction in other forums is permitted, provided the original author(s) and the copyright owner(s) are credited and that the original publication in this journal is cited, in accordance with accepted academic practice. No use, distribution or reproduction is permitted which does not comply with these terms. 